\title{
Entanglement in fermion systems
}

\author{
N. Gigena, R.Rossignoli \\ Departamento de Física-IFLP, Universidad Nacional de La Plata, C.C. 6\%, La Plata (1900), Argentina
}

\begin{abstract}
We analyze the problem of quantifying entanglement in pure and mixed states of fermionic systems with fixed number parity yet not necessarily fixed particle number. The "mode entanglement" between one single-particle level and its orthogonal complement is first considered, and an entanglement entropy for such a partition of a particular basis of the single-particle Hilbert space $\mathcal{H}$ is defined. The sum over all single-particle modes of this entropy is introduced as a measure of the total entanglement of the system with respect to the chosen basis and it is shown that its minimum over all bases of $\mathcal{H}$ is a function of the one-body density matrix. Furthermore, we show that if minimization is extended to all bases related through a Bogoliubov transformation, then the entanglement entropy is a function of the generalized one-body density matrix. These results are then used to quantify entanglement in fermion systems with four single-particle levels. For general pure states of such a system a closed expression for the fermionic concurrence is derived, which generalizes the Slater correlation measure defined in [J. Schliemann et al, Phys. Rev. A 64, 022303 (2001)], implying that "particle entanglement" may be seen as minimum "mode entanglement". It is also shown that the entanglement entropy defined before is related to this concurrence by an expression analogous to that of the two-qubit case. For mixed states of this system the convex roof extension of the previous concurrence and entanglement entropy are evaluated analytically, extending the results of previous ref. to general states.
\end{abstract}

PACS numbers: 03.67.Mn, 03.65.Ud, 05.30.Fk

\section{INTRODUCTION}

Quantum entanglement is not only one of the key features of quantum mechanics but also an essential resource in quantum information processing [1]. It plays a central role in quantum teleportation [2] and quantum computation [3]. Consequently, the understanding and quantification of this resource has become a fundamental problem in quantum information theory [4]. It has also provided deep insights into the structure of correlations and quantum phase transitions in many-body systems 5 [7].

If $\left|\Psi_{A B}\right\rangle \in \mathcal{H}_{A} \otimes \mathcal{H}_{B}$ is a pure state of a composite quantum system, its entanglement is quantified by the entanglement entropy $S\left(\rho_{A}\right)=S\left(\operatorname{Tr}_{B}\left|\Psi_{A B}\right\rangle\left\langle\Psi_{A B}\right|\right)=$ $S\left(\rho_{B}\right)$, where $S(\rho)=-\operatorname{Tr} \rho \log _{2} \rho$ is the von Neumann entropy. It is then seen that the notion of entanglement in such systems relies on the tensor product structure of its state-space [8]. In fermionic systems, however, the situation is less clear since the state-space has no longer this structure due to indistinguishability.

When generalizing the notion of entanglement to systems of indistinguishable particles 9 [18 mainly two different approaches have been taken: Mode entanglement 12 14, 19, 20 and quantum correlations/particle entanglement [9 11, 15 18, 21 23]. In the first case the parties share different modes of a given basis of the single-particle Hilbert space. Therefore, mode entanglement of a system does not remain invariant with respect to unitary transformations in the single-particle (sp) space. The second approach looks for correlations between particles and beyond antisymmetrization. In 9, 11] a fermionic analog of the Schmidt decomposition and Schmidt number was introduced to quantify entanglement in two-fermion systems, and also a fermionic "concurrence" was defined. While these measures of entanglement remain invariant under unitary transformations in the sp space, they are restricted to states with a fixed particle number, which is not the general case in fermionic systems. The same problem arises in 15, where in order to share particles between parties it is necessary to project the original state onto subspaces with definite particle number.

In this paper we first consider pure states of fermionic systems within a grand-canonical context, so the particle number is not necessarily fixed. Fermionic states with no fixed number of fermions arise, for instance, when considering the vacuum of quasiparticles defined through a Bogoliubov transformation 20, 21, 24, as well as by simply applying particle-hole transformations, such that the state is viewed as a vacuum of certain fermion operators plus particle-hole excitations. The fermion number parity of these states is nonetheless fixed, in agreement with fermionic super-selection rules 25]. The entanglement between a single fermionic mode and the remaining sp orthogonal space in such states is first considered, and an entanglement entropy is defined in order to quantify these correlations. We then propose the sum over singleparticle modes of this entropy as a measure of the total mode entanglement associated with the chosen sp basis, and show that its minimum over all single particle bases depends only on the eigenvalues of the one-body density matrix $\rho_{i j}^{\mathrm{sp}}=\left\langle c_{j}^{\dagger} c_{i}\right\rangle$, being therefore invariant under sp transformations. Furthermore, it is shown that if the minimization is extended to all quasiparticle bases, i.e., bases related through Bogoliubov transformations, the minimum entanglement entropy is just the von Neumann entropy of the generalized one-body density matrix $\rho^{\mathrm{qsp}}$, which contains in addition the pair creation 
and annihilation contractions $\left\langle c_{j}^{\dagger} c_{i}^{\dagger}\right\rangle$ and $\left\langle c_{j} c_{i}\right\rangle$. Its convex roof extension for mixed states is also introduced. This quantity allows to rigorously identify mixed states which cannot be written as convex mixtures of Slater determinants or quasiparticle vacua (or in general fermionic gaussian states 21, 22]), like thermal states of interacting fermion systems at sufficiently low temperatures, quantifying their quantum correlations.

We then focus on fermionic systems with sp space dimension 4. For general states it is shown that the minimum over all quasiparticle bases of the entanglement entropy can be written in terms of a fermionic analog of the concurrence 22, 23, 26], that reduces to the Slater correlation measure defined in 9, 11] for two-fermion states. Its convex roof extension for mixed states is also evaluated analytically, extending explicitly the results of [9] to arbitrary mixed states with fixed number parity [22]. This allows to evaluate in closed form the convex-roof extension of the previous entanglement entropy. A simple illustrative example is provided.

\section{FORMALISM}

\section{A. Single level entanglement entropy}

We start by considering a pure state $|\Psi\rangle$ of a fermion system with an $n$-dimensional single-particle (sp) Hilbert space $\mathcal{H}$. The system is described by a set of fermion annihilation and creation operators $\left\{c_{j}, c_{j}^{\dagger}\right.$, $\}$ satisfying

$$
c_{i} c_{j}+c_{j} c_{i}=0, \quad c_{i} c_{j}^{\dagger}+c_{j}^{\dagger} c_{i}=\delta_{i j}
$$

such that $\left\{c_{j}^{\dagger}|0\rangle, j=1, \ldots, n\right\}$ is an orthonormal basis of sp states $\left(|0\rangle\right.$ denotes the vacuum of the operators $\left.c_{j}\right)$. We will work within a general grand-canonical context, in which $|\Psi\rangle$ is not necessarily a state with a definite value of the fermion number $N=\sum_{j} c_{j}^{\dagger} c_{j}$. It may be, for instance, a vacuum of qusiparticle operators $a_{\nu}$, related with the $c_{j}$ 's through a Bogoliubov transformation 24]. In this case, it is a sum of pure states with different fermion numbers (see Appendix), yet having all the same number parity

$$
P=\exp \left[i \pi \sum_{j} c_{j}^{\dagger} c_{j}\right],
$$

such that $P|\Psi\rangle= \pm|\Psi\rangle$. Let us also recall that the elementary particle-hole Bogoliubov transformation

$$
c_{j} \rightarrow c_{j}^{\dagger}, \quad c_{j}^{\dagger} \rightarrow c_{j}
$$

leaves the anticommutation relations unchanged, so that formally, it is a matter of choice whether one considers the particles or the holes as the "true" fermions. We will take this basic symmetry into account in all the following correlation measures, such that they all remain invariant under the previous transformation. We will just assume that all pure states involved have a definite number parity 25], which implies $\left\langle c_{j}\right\rangle=0$ and also $\langle O\rangle=0$ for any operator $O$ which is a product of an odd number of fermion operators $c_{j}, c_{j}^{\dagger}$.

We now consider a partition $(A, B)$ of $\mathcal{H}$, where $A$ denotes the single mode or "level" $j$ and $B$ the remaining orthogonal sp space. Eq. (1) implies that the operators

$$
\Pi_{j}=c_{j}^{\dagger} c_{j}, \quad \Pi_{\bar{j}}=c_{j} c_{j}^{\dagger}, \quad \Pi_{j}+\Pi_{\bar{j}}=1,
$$

constitute a basic set of orthogonal projectors, defining a standard projective measurement on the level $j$. Accordingly, we may decompose any state $|\Psi\rangle$ as

$$
\begin{aligned}
|\Psi\rangle & =c_{j}^{\dagger} c_{j}|\Psi\rangle+c_{j} c_{j}^{\dagger}|\Psi\rangle \\
& =\sqrt{p_{j}}\left|\Psi_{j}\right\rangle+\sqrt{p_{\bar{j}}}\left|\Psi_{\bar{j}},\right\rangle
\end{aligned}
$$

where the first (second) term in (5) selects the component of $|\Psi\rangle$ where the state $j$ is occupied (empty) and $\left|\Psi_{j}\right\rangle=\frac{1}{\sqrt{p}_{j}} c_{j}^{\dagger} c_{j}|\Psi\rangle,\left|\Psi_{\bar{j}}\right\rangle=\frac{1}{\sqrt{\bar{p}_{\bar{j}}}} c_{j} c_{j}^{\dagger}|\Psi\rangle$ are the corresponding normalized states. Here $p_{j}\left(p_{\bar{j}}\right)$ is the probability of finding the level $j$ occupied (empty) in $|\Psi\rangle$ :

$$
p_{j}=\left\langle\Psi\left|c_{j}^{\dagger} c_{j}\right| \Psi\right\rangle, \quad p_{\bar{j}}=\left\langle\Psi\left|c_{j} c_{j}^{\dagger}\right| \Psi\right\rangle=1-p_{j} .
$$

For an operator $O_{A}$ depending just on $c_{j}, c_{j}^{\dagger}$ and $O_{B}$ depending just on the complementary set $\left\{c_{k}, c_{k}^{\dagger}, k \neq j\right\}$, we then obtain, assuming $P|\Psi\rangle= \pm|\Psi\rangle$,

$$
\begin{aligned}
\left\langle\Psi\left|O_{A(B)}\right| \Psi\right\rangle & =p_{j}\left\langle\Psi_{j}\left|O_{A(B)}\right| \Psi_{j}\right\rangle+p_{\bar{j}}\left\langle\Psi_{\bar{j}}\left|O_{A(B)}\right| \Psi_{\bar{j}}\right\rangle \\
& =\operatorname{tr}_{A(B)} \rho_{A(B)} O_{A(B)}
\end{aligned}
$$

where $\rho_{A}=p_{j} c_{j}^{\dagger}|0\rangle\left\langle 0\left|c_{j}+p_{\bar{j}}\right| 0\right\rangle\langle 0|$ and $\rho_{B}=$ $p_{j} c_{j}\left|\Psi_{j}\right\rangle\left\langle\Psi_{j}\left|c_{j}^{\dagger}+p_{\bar{j}}\right| \Psi_{\bar{j}}\right\rangle\left\langle\Psi_{\bar{j}}\right|$ represent reduced density operators for systems $A$ and $B$ respectively.

The entanglement between $A$ and $B$ can then be quantified by the entropy of the elementary distribution $\left\{p_{j}, p_{\bar{j}}=1-p_{j}\right\}$ :

$$
\begin{aligned}
S\left(\rho_{A}\right)=S\left(\rho_{B}\right) & =-p_{j} \log _{2} p_{j}-\left(1-p_{j}\right) \log _{2}\left(1-p_{j}\right)(9) \\
& =h\left(p_{j}\right),
\end{aligned}
$$

where $S(\rho)=-\operatorname{Tr} \rho \log _{2} \rho$ is the von Neumann entropy and $h(p)=-p \log _{2} p-(1-p) \log _{2}(p)(0 \leq h(p) \leq 1)$. This entropy remains obviously invariant after a particle-hole transformation (3). For a pure state $|\Psi\rangle$, Eq. (9) vanishes if and only if (iff) $|\Psi\rangle$ is separable with respect to this level, i.e., iff the level $j$ is either occupied $\left(p_{j}=1\right)$ or empty $\left(p_{j}=0\right)$ in $|\Psi\rangle$, such that $|\Psi\rangle=c_{j}^{\dagger} c_{j}|\Psi\rangle$ or $|\Psi\rangle=$ $c_{j} c_{j}^{\dagger}|\Psi\rangle$ respectively. Its maximum value 1 is attained for $p_{j}=1 / 2$.

\section{B. One-body entanglement entropy}

The sum

$$
S_{c}=\sum_{j} h\left(p_{j}\right)
$$


is a measure of the entanglement associated with the sp basis determined by the operators $c_{j}^{\dagger}$. Eq. (11) vanishes iff each level $j$ of this basis is disentangled from its complementary sp space, i.e., iff each level is either occupied or empty in $|\Psi\rangle$, such that $|\Psi\rangle$ is a Slater determinant in this basis: $|\Psi\rangle=c_{j_{1}}^{\dagger} \ldots c_{j_{m}}^{\dagger}|0\rangle$ for some subset of levels $\left\{j_{1}, \ldots, j_{m}\right\}$.

Eq. (11) depends on the choice of sp basis, i.e., on the choice of fermion operators $c=\left(c_{1} \ldots, c_{n}\right)^{T}$. We now consider the minimum of (11) over all sp bases of $\mathcal{H}$, i.e.,

$$
S^{\mathrm{sp}}=\underset{\boldsymbol{c}^{\prime}}{\operatorname{Min}} S_{\boldsymbol{c}^{\prime}}
$$

where $S_{c^{\prime}}=\sum_{j} h\left(p_{j}^{\prime}\right)$, with $p_{j}^{\prime}=\left\langle\Psi\left|c_{j}^{\dagger} c_{j}^{\prime}\right| \Psi\right\rangle$ and $\boldsymbol{c}^{\prime}=$ $\left(c_{1}^{\prime}, \ldots, c_{n}^{\prime}\right)^{T}$ an arbitrary set of fermion operators related with the $c_{j}$ 's through a unitary transformation:

$$
c^{\prime}=U^{\dagger} \boldsymbol{c}
$$

with $U$ a $n \times n$ unitary matrix (such that the fermionic relations (11) are preserved). Eq. (12) vanishes iff $|\Psi\rangle$ is a Slater determinant, i.e., $|\Psi\rangle=c^{\prime \dagger}{ }_{k_{1}}^{\dagger} \ldots c_{k_{m}}^{\prime \dagger}|0\rangle$ for some operators $c_{k}^{\prime}$ of the form (13). Hence, $S^{\mathrm{sp}}=0$ iff there is a sp basis where every level is disentangled from its complementary sp space.

Defining the sp density matrix $\rho^{\mathrm{sp}}=1-\left\langle\boldsymbol{c} \boldsymbol{c}^{\dagger}\right\rangle$ (with $\langle O\rangle \equiv\langle\Psi|O| \Psi\rangle)$, of elements

$$
\rho_{i j}^{\mathrm{sp}}=\left\langle c_{j}^{\dagger} c_{i}\right\rangle
$$

it is seen that the minimum (12) is reached for those operators $\boldsymbol{c}^{\prime}$ which diagonalize $\rho^{\mathrm{sp}}$, i.e., satisfying

$$
\left\langle c_{k}^{\prime \dagger} c_{l}^{\prime}\right\rangle=\left(U^{\dagger} \rho^{\mathrm{sp}} U\right)_{l k}=p_{k}^{\prime} \delta_{k l}
$$

with $p_{k}^{\prime}$ the eigenvalues of $\rho^{\mathrm{sp}}$.

Proof: Eqs. (13)-15) imply that $p_{j}=\rho_{j j}^{\mathrm{sp}}=\sum_{k}\left|U_{j k}^{2}\right| p_{k}^{\prime}$. Hence, concavity of the function $h(p)$ entails $\sum_{j} h\left(p_{j}\right) \geq$ $\sum_{j, k}\left|U_{j k}^{2}\right| h\left(p_{k}^{\prime}\right)=\sum_{k} h\left(p_{k}^{\prime}\right)$, with equality reached iff the $p_{j}$ 's are already the eigenvalues of $\rho^{\mathrm{sp}}$.

The minimum value (12) can then be expressed as

$$
S^{\mathrm{sp}}=\sum_{k} h\left(p_{k}^{\prime}\right)=\operatorname{tr} h\left(\rho^{\mathrm{sp}}\right),
$$

being now apparent that $S^{\mathrm{sp}}$ vanishes iff the eigenvalues $p_{k}^{\prime}$ are either 0 or 1 , i.e., iff $\left(\rho^{\mathrm{sp}}\right)^{2}=\rho^{\mathrm{sp}}$, a condition ensuring that $|\Psi\rangle$ is a Slater determinant 24].

Eq. (16) has in addition the obvious meaning of quantifying how mixed or "hot" is $|\Psi\rangle$ with respect to the set of all one-body operators of the form

$$
O=\sum_{i, j} o_{i j} c_{i}^{\dagger} c_{j}
$$

since their averages are completely determined just by $\rho^{\mathrm{sp}}:\langle\Psi|O| \Psi\rangle=\operatorname{tr} \rho^{\mathrm{sp}} o$. Accordingly, $S^{\mathrm{sp}}$ remains invariant under one-body unitary transformations $|\Psi\rangle \rightarrow$ $\exp (-i O)|\Psi\rangle$, with $O$ any hermitian one-body operator of form (17), since they lead to a unitary transformation of $\rho^{\mathrm{sp}}\left(\rho^{\mathrm{sp}} \rightarrow U \rho^{\mathrm{sp}} U^{\dagger}\right.$, with $\left.U=e^{-i o}\right)$ and hence do not affect its eigenvalues.

\section{Generalized one-body entanglement entropy}

A quasiparticle vacuum, like for instance a superfluid or superconducting state in the BCS approximation 24, will lead to $S^{\mathrm{sp}}>0$, since $\rho^{\mathrm{sp}}$ will be mixed, i.e., it will have eigenvalues distinct from 0 or 1 (see Appendix). If fermion quasiparticles are to be allowed, we can extend the minimization in (12) to all single quasiparticle basis, i.e.,

$$
S^{\mathrm{qsp}}=\operatorname{Min}_{\boldsymbol{a}} S_{\boldsymbol{a}}
$$

where $S_{\boldsymbol{a}}=\sum_{\nu} h\left(\left\langle a_{\nu}^{\dagger} a_{\nu}\right\rangle\right)$ and $\boldsymbol{a}$ denotes a set of fermion operators $a_{\nu}$ linearly related to the original operators $c_{j}$, $c_{j}^{\dagger}$ through a general Bogoliubov transformation 24]:

$$
a_{\nu}=\sum_{j} \bar{U}_{j \nu} c_{j}+V_{j \nu} c_{j}^{\dagger}
$$

Eq. (19) can be written as

$$
\left(\begin{array}{c}
\boldsymbol{a} \\
\boldsymbol{a}^{\dagger}
\end{array}\right)=\mathcal{W}^{\dagger}\left(\begin{array}{c}
\boldsymbol{c} \\
\boldsymbol{c}^{\dagger}
\end{array}\right), \mathcal{W}=\left(\begin{array}{cc}
U & V \\
\bar{V} & \bar{U}
\end{array}\right),
$$

where the $2 n \times 2 n$ matrix $\mathcal{W}$ should be unitary (i.e. $\left.U U^{\dagger}+V V^{\dagger}=1, \quad U V^{T}+V U^{T}=0\right)$ in order that the operators $a_{\nu}, a_{\mu}^{\dagger}$ fulfill the fermionic anticommutation relations (1).

One should then consider the extended $2 n \times 2 n$ density matrix

$$
\rho^{\mathrm{qsp}}=1-\left\langle\left(\begin{array}{c}
\boldsymbol{c} \\
\boldsymbol{c}^{\dagger}
\end{array}\right)\left(\begin{array}{ll}
\boldsymbol{c}^{\dagger} & \boldsymbol{c}
\end{array}\right)\right\rangle=\left(\begin{array}{cc}
\rho^{\mathrm{sp}} & \kappa \\
-\bar{\kappa} & 1-\bar{\rho}^{\mathrm{sp}}
\end{array}\right)
$$

where $\kappa$ is an $n \times n$ antisymmetric matrix containing the pair annihilation averages

$$
\kappa_{i j}=\left\langle c_{j} c_{i}\right\rangle
$$

with $-\bar{\kappa}_{i j}=\left\langle c_{j}^{\dagger} c_{i}^{\dagger}\right\rangle$ and $\left(1-\bar{\rho}^{\mathrm{sp}}\right)_{i j}=\left\langle c_{j} c_{i}^{\dagger}\right\rangle$. Eq. 210 is a hermitic matrix which can always be diagonalized by a suitable transformation (20), such that

$$
1-\left\langle\left(\begin{array}{c}
\boldsymbol{a} \\
\boldsymbol{a}^{\dagger}
\end{array}\right)\left(\begin{array}{ll}
\boldsymbol{a} & \boldsymbol{a}^{\dagger}
\end{array}\right)\right\rangle=\mathcal{W}^{\dagger} \rho^{\mathrm{qsp}} \mathcal{W}=\left(\begin{array}{cc}
f & 0 \\
0 & 1-f
\end{array}\right),
$$

with $f_{\mu \nu}=f_{\nu} \delta_{\mu \nu}$ and $f_{\nu}, 1-f_{\nu}$ the eigenvalues of $\rho^{\text {qsp }}$ (which always come in pairs $\left(f_{\nu}, 1-f_{\nu}\right)$, with $f_{\nu} \in[0,1]$ ), entailing

$$
\left\langle a_{\nu}^{\dagger} a_{\mu}\right\rangle=\delta_{\mu \nu} f_{\nu}, \quad\left\langle a_{\mu} a_{\nu}\right\rangle=0 .
$$

It can then be easily shown that the minimum (18) is

$$
\begin{aligned}
S^{\mathrm{qsp}} & =-\sum_{\nu} f_{\nu} \log _{2} f_{\nu}+\left(1-f_{\nu}\right) \log _{2}\left(1-f_{\nu}\right) \\
& =-\operatorname{tr}^{\prime} \rho^{\mathrm{qsp}} \log _{2} \rho^{\mathrm{qsp}} .
\end{aligned}
$$

where $\operatorname{tr}^{\prime}$ denotes the trace in the extended sp space.

Proof: Since both $p_{j}=\left\langle c_{j}^{\dagger} c_{j}\right\rangle$ and $1-p_{j}$ are the diagonal 
elements of $\rho^{\mathrm{qsp}}$, denoting with $q_{j}$ and $\lambda_{\nu}$ the full set of diagonal elements and eigenvalues of $\rho^{\mathrm{qsp}}$, we obtain $q_{j}=\sum_{\nu}\left|\mathcal{W}_{j \nu}^{2}\right| \lambda_{\nu}$ and hence, due to the concavity of $f(p)=-p \log _{2} p, S_{c}=\sum_{j} f\left(q_{j}\right) \geq \sum_{j, \nu}\left|\mathcal{W}_{j \nu}^{2}\right| f\left(\lambda_{\nu}\right)=$ $\sum_{\nu} f\left(\lambda_{\nu}\right)=S^{\mathrm{qsp}}$.

Eq. (26) vanishes iff $f_{\nu}$ is either 0 or 1 for all $\nu$, i.e., iff $|\Psi\rangle$ is a particle or quasiparticle Slater determinant. By an elementary particle-hole transformation we can always change such state to a quasiparticle vacuum, so that we can say $S^{\mathrm{qsp}}=0$ iff $|\Psi\rangle$ is a quasiparticle vacuum. In other words, $S^{\mathrm{qsp}}=0$ iff there is a single quasiparticle basis where every level is disentangled from the rest.

Eq. (18) also measures the mixedness of $|\Psi\rangle$ with respect to the set of all generalized one-body operators, of the form

$$
\begin{aligned}
O & =\sum_{i, j} o_{i j}^{11} c_{i}^{\dagger} c_{j}+\frac{1}{2}\left(o_{i j}^{20} c_{i} c_{j}+o_{i j}^{02} c_{i}^{\dagger} c_{j}^{\dagger}\right)-\frac{1}{2} \operatorname{tr} o^{11} \\
& =\frac{1}{2}\left(\boldsymbol{c}^{\dagger} \boldsymbol{c}\right) \mathcal{O}\left(\begin{array}{c}
\boldsymbol{c} \\
\boldsymbol{c}^{\dagger}
\end{array}\right), \mathcal{O}=\left(\begin{array}{cc}
o^{11} & o^{02} \\
o^{20} & -\left(o^{11}\right)^{T}
\end{array}\right)
\end{aligned}
$$

i.e., general quadratic functions of $\boldsymbol{c}, \boldsymbol{c}^{\dagger}$ (the constant term in (27) is just added for convenience), since their averages are completely determined by $\rho^{\mathrm{qsp}}$ :

$$
\langle\Psi|O| \Psi\rangle=\operatorname{tr}\left[\rho^{\mathrm{sp}} o^{11}-\frac{1}{2} o^{11}+\frac{1}{2}\left(\kappa o^{20}-\bar{\kappa} O^{02}\right)\right]=\frac{1}{2} \operatorname{tr}^{\prime} \rho^{\mathrm{qsp}} \mathcal{O}
$$

The present scheme allows then to properly treat states which do not have a definite fermion number and lead to non-zero contractions $\left\langle c_{i} c_{j}\right\rangle$. The whole formalism becomes then strictly invariant under arbitrary particle hole transformations (3) applied to some subset of levels, which will move elements from $\rho^{\mathrm{sp}}$ to $\kappa$ and viceversa, but which will not alter the spectrum of $\rho^{\mathrm{qsp}}$. The latter remains actually invariant under arbitrary quasiparticle unitary transformations $|\Psi\rangle \rightarrow \exp [-i O]|\Psi\rangle$, where $O$ is an hermitian generalized one-body operator of the form (27), since they just lead to a unitary transformation of $\rho^{\mathrm{qsp}}$, i.e., $\rho^{\mathrm{qsp}} \rightarrow \mathcal{W} \rho^{\mathrm{qsp}} \mathcal{W}^{\dagger}$, with $\mathcal{W}=e^{-i \mathcal{O}}$.

We notice that a transformation $a_{\nu} \leftrightarrow a_{\nu}^{\dagger}$ obviously changes $f_{\nu} \leftrightarrow 1-f_{\nu}$, so that there is no unique way to select which of the eigenvalues of $\rho^{\text {qsp }}$ will be the $f_{\nu}$ 's or the $1-f_{\nu}^{\prime} s$. One can choose the $f_{\nu}$ 's as the lowest eigenvalues (such that $|\Psi\rangle$ becomes a quasiparticle vacuum when $\left.S^{\mathrm{qsp}}=0\right)$, but it is also possible to set $\operatorname{Det}[U] \neq 0$, which ensures that the vacuum of the $a_{\nu}$ has the same number parity as $|0\rangle$ (Eq. A1). These choices do not affect the entropy $S^{\mathrm{qsp}}$. We also remark that the maximally entangled state, i.e., that with maximum $S^{\mathrm{qsp}}$, corresponds to the exceptional case $f_{\nu}=1 / 2 \forall \nu$, where $S^{\text {qsp }}=n$ and $\rho^{\mathrm{qsp}}=I_{2 n} / 2$ becomes proportional to the identity matrix, remaining then invariant under any Bogoliubov transformation.

\section{Generalized entropic inequalities and quadratic entropy}

From their definitions, it follows that the entropies (11), (16) and (26) satisfy the inequality chain

$$
S_{c} \geq S^{\mathrm{sp}} \geq S^{\mathrm{qsp}} .
$$

Eq. (30) actually holds for more general entropic forms. If $\tilde{\rho}^{\mathrm{sp}}=\left(\begin{array}{cc}\rho^{\mathrm{sp}} & 0 \\ 0 & 1-\rho^{\mathrm{sp}}\end{array}\right)$ is the extended $\rho^{\mathrm{sp}}$ and $\tilde{\rho}_{d}^{\mathrm{sp}}$ the diagonal of $\tilde{\rho}^{\text {sp }}$, we obtain, with the same previous arguments,

$$
S_{f}\left(\tilde{\rho}_{d}^{\mathrm{sp}}\right) \geq S_{f}\left(\tilde{\rho}^{\mathrm{sp}}\right) \geq S_{f}\left(\rho^{\mathrm{qsp}}\right),
$$

where

$$
S_{f}(\rho)=\operatorname{tr} f(\rho),
$$

with $f:[0,1] \rightarrow \mathbb{R}$ a strictly concave function satisfying $f(0)=f(1)=0$, represents a generalized entropic form 27, 28. Moreover, these matrices fulfill the majorization relation 29.

$$
\tilde{\rho}_{d}^{\mathrm{sp}} \prec \tilde{\rho}^{\mathrm{sp}} \prec \rho^{\mathrm{qsp}}
$$

where $\rho \prec \rho^{\prime}$ means here $\sum_{i=1}^{j} \lambda_{i} \leq \sum_{i=1}^{j} \lambda_{i}^{\prime}$ for $j=$ $1, \ldots, 2 n-1$, with $\lambda_{i}, \lambda_{i}^{\prime}$ the eigenvalues of $\rho$ and $\rho^{\prime}$ sorted in decreasing order, since the sorted set of diagonal elements in an orthonormal basis of an hermitian operator are always majorized by the sorted set of its eigenvalues 29. Eq. (33) allows to extend (31) to any Schur-concave function 29] of the extended density matrices.

A particularly useful example, which will play an important role in the next section, is the quadratic entropy $S_{2}(\rho)$ (also denoted as linear entropy), obtained for $f(p)=2 p(1-p)$ :

$$
\begin{aligned}
S_{2}\left(\rho^{\mathrm{qsp}}\right) & =2 \operatorname{tr}^{\prime}\left[\rho^{\mathrm{qsp}}\left(1-\rho^{\mathrm{qsp}}\right)\right] \\
& =4 \operatorname{tr}\left[\rho^{\mathrm{sp}}\left(1-\rho^{\mathrm{sp}}\right)-\kappa^{\dagger} \kappa\right] \\
& =4 \sum_{\nu} f_{\nu}\left(1-f_{\nu}\right),
\end{aligned}
$$

where the factor 2 has been chosen such that its maximum value for a single level is 1 . Unlike the von Neumman entropy (26), $S_{2}\left(\rho^{\mathrm{qsp}}\right)$ can be evaluated just by taking the trace in (34), without explicit knowledge of the eigenvalues $f_{\nu}$ of $\rho^{\mathrm{qsp}}$. Yet, like $S^{\mathrm{qsp}}$, it is nonnegative and vanishes iff $|\Psi\rangle$ is a quasiparticle vacuum or Slater Determinant. Eq. (31) implies in particular $\sum_{j} p_{j}\left(1-p_{j}\right) \geq \sum_{k} p_{k}^{\prime}\left(1-p_{k}^{\prime}\right) \geq \sum_{\nu} f_{\nu}\left(1-f_{\nu}\right)$.

\section{E. Mixed states}

Let us now consider mixed fermion states, assumed as convex mixtures of pure states with definite number parity, i.e.,

$$
\rho=\sum_{i} q_{i}\left|\Psi_{i}\right\rangle\left\langle\Psi_{i}\right|,
$$


where $q_{i} \geq 0, \sum_{i} q_{i}=1$ and $P\left|\Psi_{i}\right\rangle= \pm\left|\Psi_{i}\right\rangle$, such that $[\rho, P]=0$. We can define an entanglement measure for these mixed states in a way similar to the entanglement of formation 30, 31, through the convex roof extension of $S^{\text {qsp }}$,

$$
E^{\mathrm{qsp}}(\rho)=\underset{\left\{q_{i}^{\prime},\left|\Psi_{i}^{\prime}\right\rangle\right\}}{\operatorname{Min}} \sum_{i} q_{i}^{\prime} S^{\mathrm{qsp}}\left(\left|\Psi_{i}^{\prime}\right\rangle\right)
$$

where $\rho=\sum_{i} q_{i}^{\prime}\left|\Psi_{i}^{\prime}\right\rangle\left\langle\Psi_{i}^{\prime}\right|, q_{i}^{\prime} \geq 0$, and the minimization is over all decompositions of $\rho$ as convex mixtures of pure states, assumed again of definite number parity. Eq. (37) vanishes iff $\rho$ is a convex mixture of particle or quasiparticle Slater determinants, i.e., of suitable quasiparticle vacua, and reduces to $S^{\mathrm{qsp}}$ for pure states. This quantity will be evaluated exactly in the particular system of the next section.

As a general application of $E^{\mathrm{qsp}}$, let us consider an interacting fermion system at finite temperature $T$. For attractive two-body couplings, the static path approximation 32, 33. will lead to a classically correlated density operator $\rho_{\mathrm{SPA}}$, which is a convex mixture of (noncommuting) thermal states diagonal in a basis of particle or quasiparticle Slater determinants, associated with different values of the running effective order parameters. Hence, $E^{\mathrm{qsp}}\left(\rho_{\mathrm{SPA}}\right)=0$, in agreement with the fact that $\rho_{\mathrm{SPA}}$ contains just static fluctuations around mean field. Such correlated but still unentangled approximation can be derived from the auxiliary field path integral representation 34, and becomes exact at sufficiently high $T$ 33]. Its breakdown at low $T$ reflects the onset of entanglement, i.e., of a finite value of $E^{\mathrm{qsp}}(\rho)$. Eq. (37) defines a limit temperature $T_{L}$ above which $E^{\mathrm{qsp}}=0$. Mixtures of fermionc gaussian states are also important in noisy fermionic quantum computation models [22, 35].

\section{THE CASE OF FOUR SINGLE PARTICLE LEVELS}

We will now examine in detail the special case of a fermion system with single particle space dimension $n=4$. This is the lowest dimension where non-trivial fermionic entanglement arises, i.e., where $S^{\mathrm{qsp}}$ can be non-zero, as will be verified. We will extend the results of [9, which considered just pure or mixed states with a definite fermion number, to general states which do not necessarily have a definite fermion number, yet still have a definite number parity $P$ (see also 22,23$]$ ). This sp space can accommodate 8 linearly independent pure states of the same number parity, so that the Hilbert space dimension for fixed $P$ is 8 .

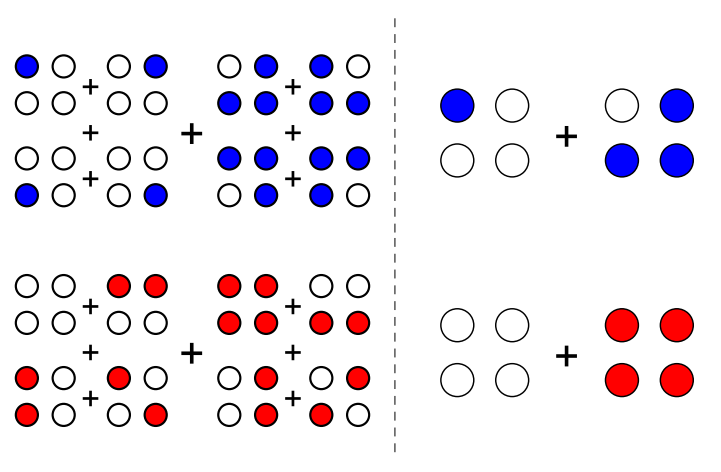

FIG. 1. (Color online) Schematic representation of pure fermion states with odd (top) or even (bottom) number parity. A general state with definite number parity is a superposition of the 8 states indicated on the left of the dashed vertical line, where a full disk indicates an occupied level. In the normal form ( $\rho^{\mathrm{qsp}}$ diagonal), obtained after a suitable Bogoliubov transformation, it can be reduced to the superposition of two states like those indicated on the right. The state is entangled (in the sense of not being a quasiparticle vacuum or Slater Determinant) iff the product $C$ (Eq. (43) ) of the coefficients of the left and right groups of four states is non-zero, implying nonzero weight for both states of the normal representation.

\section{A. Pure states}

\section{Odd parity states}

We first consider pure states $|\Psi\rangle$ of this system with odd number parity: $P|\Psi\rangle=-|\Psi\rangle$. These states are then linear combinations of single fermion states and threefermion states, so a general odd state can be written as (Fig. 11 top)

$$
|\Psi\rangle=\sum_{i=1}^{4}\left(\alpha_{i} c_{i}^{\dagger}|0\rangle+\bar{\beta}_{i} c_{i}|\overline{0}\rangle\right),
$$

where $|\overline{0}\rangle=c_{1}^{\dagger} c_{2}^{\dagger} c_{3}^{\dagger} c_{4}^{\dagger}|0\rangle$ is the completely occupied state and $|\boldsymbol{\alpha}|^{2}+|\boldsymbol{\beta}|^{2}=1$, with $\boldsymbol{\alpha}, \boldsymbol{\beta}$ four dimensional complex vectors. It is easily seen that the single hole states $c_{i}|\overline{0}\rangle$ are

$$
c_{i}|\overline{0}\rangle=\frac{1}{3 !} \sum_{j, k, l} \epsilon_{i j k l} c_{j}^{\dagger} c_{k}^{\dagger} c_{l}^{\dagger}|0\rangle,
$$

where $\epsilon_{i j k l}$ denotes the completely antisymmetric LeviCivita tensor in dimension 4 . The elements of the generalized one-body density matrix (21) are then given by

$$
\begin{aligned}
& \rho_{i j}^{\mathrm{sp}}=\left\langle c_{j}^{\dagger} c_{i}\right\rangle=\alpha_{i} \bar{\alpha}_{j}-\beta_{i} \bar{\beta}_{j}+|\boldsymbol{\beta}|^{2} \delta_{i j}, \\
& \kappa_{i j}=\left\langle c_{j} c_{i}\right\rangle=\sum_{k, l} \epsilon_{i j k l} \bar{\alpha}_{l} \bar{\beta}_{k},
\end{aligned}
$$

i.e., $\rho^{\text {sp }}=\boldsymbol{\alpha} \boldsymbol{\alpha}^{\dagger}-\boldsymbol{\beta} \boldsymbol{\beta}^{\dagger}+|\boldsymbol{\beta}|^{2} I_{4}$. We now show that the ensuing eigenvalues $f_{\nu}$ of the $8 \times 8$ matrix $\rho^{\mathrm{qsp}}$ are four- 
fold degenerate and given by

$$
f_{ \pm}=\frac{1 \pm \sqrt{1-C^{2}(|\Psi\rangle)}}{2}
$$

where $C(|\Psi\rangle)$ is fully determined by the $S_{2}$ entropy (34),

$$
\begin{aligned}
C(|\Psi\rangle) & =\sqrt{S_{2}\left(\rho^{\mathrm{qsp}}\right) / 4}=\sqrt{\operatorname{tr}\left[\rho^{\mathrm{sp}}\left(I_{4}-\rho^{\mathrm{sp}}\right)-\kappa^{\dagger} \kappa\right]} \\
& =2\left|\boldsymbol{\beta}^{\dagger} \boldsymbol{\alpha}\right|=2\left|\sum_{i=1}^{4} \bar{\beta}_{i} \alpha_{i}\right|,
\end{aligned}
$$

and plays the role of a pure state fermionic concurrence. It satisfies $0 \leq C \leq 1$, and as will be seen in the next subsection, it is the generalization of the Slater correlation measure defined in 9, 11] for two fermion states. It also coincides with the quadratic invariant derived in 23] using a spinors classification based approach. The entanglement entropy (25) becomes

$$
S^{\mathrm{qsp}}=4 h\left(f_{+}\right)=-4\left(f_{+} \log _{2} f_{+}+f_{-} \log _{2} f-\right) .
$$

Proof. We first consider a unitary transformation $c \rightarrow$ $U c$ of the operators $c_{j}$, such that

$$
\boldsymbol{\alpha} \rightarrow U^{\dagger} \boldsymbol{\alpha}, \boldsymbol{\beta} \rightarrow \operatorname{Det}\left[U^{\dagger}\right] U^{\dagger} \boldsymbol{\beta},
$$

in (38), which does not affect the value of $C(|\Psi\rangle)$ (Eq. (43)). By choosing an orthonormal basis of $\mathbb{C}^{4}$ such that the original vectors $\boldsymbol{\alpha}$ and $\boldsymbol{\beta}$ are generated by the first two elements (for instance, $\boldsymbol{e}_{1} \propto \boldsymbol{\alpha}$ and $\boldsymbol{e}_{2} \propto$ $\left.\boldsymbol{\beta}-\left(\boldsymbol{\alpha}^{\dagger} \boldsymbol{\beta}\right) \boldsymbol{\alpha} /|\boldsymbol{\alpha}|^{2}\right)$, we can use this first transformation to set $\alpha_{3}=\alpha_{4}=0, \beta_{3}=\beta_{4}=0$ in the new basis. In this case, Eqs. (40)-41) lead to

$$
\begin{aligned}
& \rho^{\mathrm{sp}}=\left(\begin{array}{cccc}
\left|\alpha_{1}\right|^{2}+\left|\beta_{2}\right|^{2} & \alpha_{1} \bar{\alpha}_{2}-\beta_{1} \bar{\beta}_{2} & 0 & 0 \\
\alpha_{2} \bar{\alpha}_{1}-\beta_{2} \bar{\beta}_{1} & \left|\alpha_{2}\right|^{2}+\left|\beta_{1}\right|^{2} & 0 & 0 \\
0 & 0 & |\boldsymbol{\beta}|^{2} & 0 \\
0 & 0 & 0 & |\boldsymbol{\beta}|^{2}
\end{array}\right), \\
& \kappa=\left(\begin{array}{cccc}
0 & 0 & 0 & 0 \\
0 & 0 & 0 & 0 \\
0 & 0 & 0 & \bar{\alpha}_{2} \bar{\beta}_{1}-\bar{\alpha}_{1} \bar{\beta}_{2} \\
0 & 0 & \bar{\alpha}_{1} \bar{\beta}_{2}-\bar{\alpha}_{2} \bar{\beta}_{1} & 0
\end{array}\right) .
\end{aligned}
$$

It is then seen that the diagonalization of $\rho^{\mathrm{qsp}}$ is achieved through i) a unitary transformation of the operators $c_{1}$, $c_{2}$,

$$
c_{1}=u a_{1}+v a_{2}, \quad c_{2}=-\bar{v} a_{1}+u a_{2},
$$

with $\underset{|v|}{u}=\sqrt{\frac{f_{+}-f_{-} \pm 2 \epsilon}{2\left(f_{+}-f_{-}\right)}}$and $\epsilon=\left|\alpha_{1}^{2}\right|+\left|\beta_{2}^{2}\right|-\frac{1}{2}$, which diagonalizes the first $2 \times 2$ block of $\rho^{\mathrm{sp}}$ and $1-\bar{\rho}^{\mathrm{sp}}$, plus ii) a Bogoliubov transformation of the operators $c_{3}, c_{4}$,

$$
c_{3}=u^{\prime} a_{3}+v^{\prime} a_{4}^{\dagger}, \quad c_{4}^{\dagger}=-\bar{v}^{\prime} a_{3}+u^{\prime} a_{4}^{\dagger},
$$

with $\frac{u^{\prime}}{\left|v^{\prime}\right|}=\sqrt{\frac{f_{+}-f_{-} \pm 2 \epsilon^{\prime}}{2\left(f_{+}-f_{-}\right)}}$and $\epsilon^{\prime}=\left|\boldsymbol{\beta}^{2}\right|-\frac{1}{2}$, which diagonalizes the rest of $\rho^{\text {qsp }}$, comprising again two $2 \times 2$ blocks $\left(\begin{array}{c}|\boldsymbol{\beta}|^{2} \pm \kappa_{34} \\ \pm \bar{\kappa}_{34}|\boldsymbol{\alpha}|^{2}\end{array}\right)$. These four $2 \times 2$ blocks have all trace 1 and determinant $C^{2}(|\Psi\rangle) / 4$, leading then to the same eigenvalues $f_{ \pm}$of Eq. (42) (a $2 \times 2$ matrix with trace $t$ and determinant $d$ has eigenvalues $\left.\frac{t \pm \sqrt{t^{2}-4 d}}{2}\right)$.

Note from (477) that if $\rho^{\mathrm{qsp}}$ is diagonal ( $\rho^{\mathrm{sp}}$ diagonal and $\kappa=0)$ and $C(|\Psi\rangle)<1$, then necessarily $\alpha_{2}=\beta_{2}=0$ or $\alpha_{1}=\beta_{1}=0$ in (47). This implies that after the previous transformations, $|\Psi\rangle$ can be written in the normal form (top right scheme in Fig. 1)

$$
|\Psi\rangle=\alpha^{\prime} a_{1}^{\dagger}\left|0_{\boldsymbol{a}}\right\rangle+\bar{\beta}^{\prime} a_{1}\left|\overline{0}_{\boldsymbol{a}}\right\rangle,
$$

i.e., $\boldsymbol{\beta}^{\prime} \propto \boldsymbol{\alpha}^{\prime}$, with $\left|0_{\boldsymbol{a}}\right\rangle$ the vacuum of the $\boldsymbol{a}$ operators, $\left|\overline{0}_{\boldsymbol{a}}\right\rangle=a_{1}^{\dagger} a_{2}^{\dagger} a_{3}^{\dagger} a_{4}^{\dagger}\left|0_{\boldsymbol{a}}\right\rangle$, and $\left|\alpha^{\prime}\right|^{2}=f_{+},\left|\beta^{\prime}\right|^{2}=f_{-}$if $\left|\alpha^{\prime}\right| \geq$ $\left|\beta^{\prime}\right|$, such that $C(|\Psi\rangle)=2\left|\alpha^{\prime} \bar{\beta}^{\prime}\right|$. This state leads to

$$
\rho_{a}^{\mathrm{qsp}}=1-\left\langle\left(\begin{array}{c}
\boldsymbol{a} \\
\boldsymbol{a}^{\dagger}
\end{array}\right)\left(\begin{array}{ll}
\boldsymbol{a}^{\dagger} & \boldsymbol{a}
\end{array}\right)\right\rangle=\left(\begin{array}{cccc}
\left|\alpha^{\prime}\right|^{2} & 0 & 0 & 0 \\
0 & \left|\beta^{\prime}\right|^{2} I_{3} & 0 & 0 \\
0 & 0 & \left|\beta^{\prime}\right|^{2} & 0 \\
& 0 & 0 & \left|\alpha^{\prime}\right|^{2} I_{3}
\end{array}\right) .
$$

On the other hand, in the maximally entangled case $C(|\Psi\rangle)=1, f_{ \pm}=1 / 2$ and $\rho^{\mathrm{qsp}}=I_{8} / 2$ in any basis, i.e., after any Bogoliubov transformation. In this case $\boldsymbol{\beta}=e^{i \phi} \boldsymbol{\alpha}$, with $|\boldsymbol{\alpha}|=|\boldsymbol{\beta}|=1 / \sqrt{2}$, and the form (50) is obtained just by choosing $\boldsymbol{e}_{1}$ in the direction of $\boldsymbol{\alpha}$.

It is apparent that if $\boldsymbol{\beta}=\mathbf{0}$ in (38), $|\Psi\rangle$ can be written a single fermion state $a_{1}^{\dagger}|0\rangle$, where $a_{1}^{\dagger}=\sum_{i} \alpha_{i} c_{i}^{\dagger}$. Similarly, if $\boldsymbol{\alpha}=\mathbf{0}|\Psi\rangle$ can be written a single hole state $a_{1}|\overline{0}\rangle$, with $a_{1}=\sum_{i} \bar{\beta}_{i} c_{i}$. Accordingly, $C(|\Psi\rangle)=0$ in these cases. The vanishing of $C(|\Psi\rangle)$ for nonzero but orthogonal $\boldsymbol{\alpha}$ and $\boldsymbol{\beta}$ (Eq. (43)) generalizes the previous result, showing that in this case $|\Psi\rangle$ can still be written as single quasiparticle $\left(\beta^{\prime}=0\right)$ or quasihole $\left(\alpha^{\prime}=0\right)$ after a suitable Bogoliubov transformation of the original operators. This includes the three level case where, for instance, the fourth level is empty, which implies $\alpha_{4}=0$ and $\beta_{i}=0$ for $i=1,2,3$, leading necessarily to $\boldsymbol{\beta}^{\dagger} \boldsymbol{\alpha}=0$.

We also mention that the four eigenvalues of $\rho^{\mathrm{sp}}$ in Eq. (46) are $f_{ \pm}$and $|\boldsymbol{\beta}|^{2}$, the latter two-fold degenerate. Since $C(|\Psi\rangle) \leq 2|\boldsymbol{\alpha}||\boldsymbol{\beta}|$,

$$
f_{+} \geq \frac{1+\sqrt{1-4|\boldsymbol{\alpha}|^{2}|\boldsymbol{\beta}|^{2}}}{2}=\operatorname{Max}\left[|\boldsymbol{\alpha}|^{2},|\boldsymbol{\beta}|^{2}\right],
$$

being then verified that the eigenvalues of $\rho^{\mathrm{sp}}$ are majorized by those of $\rho^{\mathrm{qsp}}$ and hence, that $S^{\mathrm{sp}} \geq S^{\mathrm{qsp}}$, $S_{2}^{\mathrm{sp}} \geq S_{2}^{\mathrm{qsp}}=4 C^{2}(|\Psi\rangle)$.

Dualization. Eqs. (38) and (43) indicate that the state $c_{i}|\overline{0}\rangle$ plays the role of partner or dual of the state $c_{i}^{\dagger}|0\rangle$. We may obtain the partner state with the hermitian operator

$$
T=-\frac{1}{3 !} \sum_{i, j, k, l} \varepsilon_{i j k l}\left[c_{i}^{\dagger} c_{j}^{\dagger} c_{k}^{\dagger} c_{l}+c_{i}^{\dagger} c_{j} c_{k} c_{l}\right]
$$

such that for $i=1, \ldots, 4, T c_{i}^{\dagger}|0\rangle=c_{i}|\overline{0}\rangle, T c_{i}|\overline{0}\rangle=c_{i}^{\dagger}|0\rangle$. We can then express Eq. (43) as

$$
C(|\Psi\rangle)=|\langle\tilde{\Psi} \mid \Psi\rangle|, \quad|\tilde{\Psi}\rangle=T|\Psi\rangle^{*}
$$


where $|\Psi\rangle^{*}=\sum_{j} \bar{\alpha}_{i} c_{i}^{\dagger}|0\rangle+\beta_{i} c_{i}|\overline{0}\rangle$ denotes the "conjugated" state in this basis. Note that the $8 \times 8$ matrix that represents $T$ in the basis $\left(c_{1}^{\dagger}|0\rangle, \ldots, c_{4}^{\dagger}|0\rangle, c_{1}|\overline{0}\rangle, \ldots, c_{4}|\overline{0}\rangle\right)$ is just

$$
T=\left(\begin{array}{cc}
0 & I_{4} \\
I_{4} & 0
\end{array}\right)
$$

A generalization of (51) for higher dimensions is considered in 23].

\section{Even parity states}

We now consider pure states of even number parity, $P|\Psi\rangle=|\Psi\rangle$. They can be obtained, for instance, by changing a particle for a hole in the odd-parity states. An even state is then a linear combination of the eight states shown in the bottom plots of Fig. 11 comprising the vacuum $|0\rangle$, six two-fermion states and the completely full state $|\overline{0}\rangle=c_{1}^{\dagger} c_{2}^{\dagger} c_{3}^{\dagger} c_{4}^{\dagger}|0\rangle$. We can write this state as

$$
|\Psi\rangle=\alpha_{1}|0\rangle-\bar{\beta}_{1}|\overline{0}\rangle+\sum_{j=2}^{4} \alpha_{j} c_{j}^{\dagger} c_{1}^{\dagger}|0\rangle+\bar{\beta}_{j} c_{1} c_{j}|\overline{0}\rangle
$$

which is just Eq. (38) with the replacements $c_{1}^{\dagger} \leftrightarrow c_{1}$ and $|0\rangle \leftrightarrow c_{1}^{\dagger}|0\rangle$, implying $|\overline{0}\rangle \leftrightarrow-c_{1}|\overline{0}\rangle$. Notice that

$$
c_{1} c_{j}|\overline{0}\rangle=\frac{1}{2 !} \sum_{k, l} \epsilon_{j 1 k l} c_{k}^{\dagger} c_{l}^{\dagger}|0\rangle .
$$

In this notation, the eigenvalues of $\rho^{\mathrm{qsp}}$ are then given by Eq. (42) with the same expression (43) for $C(|\Psi\rangle)$. The entanglement entropy $S^{\mathrm{qsp}}$ is given again by Eq. (44). Notice, however, the minus sign in the term associated with $\bar{\beta}_{1}$. The expression (43) reduces to that of [9] for the case of two-fermion states $\left(\alpha_{1}=\beta_{1}=0\right)$.

The state (54) is then a Slater Determinant or quasiparticle vacuum iff $C(|\Psi\rangle)=0$. As a check, the quasiparticle vacuum A1 corresponds in the present case to

$$
\begin{aligned}
\boldsymbol{\alpha} & \propto\left(1, T_{21}, T_{31}, T_{41}\right), \\
\overline{\boldsymbol{\beta}} & \propto\left(-T_{21} T_{43}-T_{31} T_{24}-T_{41} T_{32}, T_{43}, T_{24}, T_{32}\right),
\end{aligned}
$$

being verified that $\sum_{i=1}^{4} \bar{\beta}_{i} \alpha_{i}=0$. It is also seen that in the three-level case (i.e., level 4 empty, implying $\alpha_{4}=0$ and $\beta_{j}=0$ for $\left.j=1,2,3\right) C(|\Psi\rangle)$ is always zero.

The normal form (50) becomes here

$$
|\Psi\rangle=\alpha^{\prime}\left|0_{a}\right\rangle-\bar{\beta}^{\prime}\left|\overline{0}_{a}\right\rangle
$$

i.e., a superposition of the vacuum and the maximally occupied state (bottom right scheme in Fig. 10 for the diagonalizing quasiparticle operators. Of course, after a trivial particle hole transformation $a_{j} \leftrightarrow a_{j}^{\dagger}$ for $j=1,2$, we may always rewrite (57) as a sum of two two-fermion states, i.e.,

$$
|\Psi\rangle=\alpha^{\prime} a_{2}^{\dagger} a_{1}^{\dagger}\left|0_{\boldsymbol{a}}\right\rangle+\bar{\beta}^{\prime} a_{4}^{\dagger} a_{3}^{\dagger}\left|0_{\boldsymbol{a}}\right\rangle
$$

which extends the results of [9] valid for two-fermion states to arbitrary definite parity states.

The dualization operator (51) takes here the form

$$
T=-c_{1}^{\dagger} c_{2}^{\dagger} c_{3}^{\dagger} c_{4}^{\dagger}-c_{4} c_{2} c_{3} c_{1}-\frac{1}{4} \sum_{i, j, k, l} \epsilon_{i j k l} c_{i}^{\dagger} c_{j}^{\dagger} c_{k} c_{l},
$$

which satisfies

$T|0\rangle=-|\overline{0}\rangle, T|\overline{0}\rangle=-|0\rangle, T c_{i}^{\dagger} c_{j}^{\dagger}|0\rangle=\frac{1}{2} \sum_{k, l} \epsilon_{i j k l} c_{k}^{\dagger} c_{l}^{\dagger}|0\rangle$,

i.e., $T c_{i}^{\dagger} c_{1}^{\dagger}|0\rangle=c_{1} c_{i}|\overline{0}\rangle, \quad T c_{1} c_{i}|\overline{0}\rangle=c_{i}^{\dagger} c_{1}^{\dagger}|0\rangle$. It is represented in the special basis $\left\{|0\rangle, c_{2}^{\dagger} c_{1}^{\dagger}|0\rangle\right.$, $\left.c_{3}^{\dagger} c_{1}^{\dagger}|0\rangle, c_{4}^{\dagger} c_{1}^{\dagger}|0\rangle,-|\overline{0}\rangle, c_{4}^{\dagger} c_{3}^{\dagger}|0\rangle, c_{2}^{\dagger} c_{4}^{\dagger}|0\rangle, c_{3}^{\dagger} c_{2}^{\dagger}|0\rangle\right\}$ by the same matrix (53). We can then write again $C(|\Psi\rangle)$ in the form (52). If $\alpha_{1}=\beta_{1}=0$, the ensuing expression reduces to that of [9].

The two-fermion states considered in [9, 11] are only a particular case of the more general even states (54). For two fermion states the contractions $\left\langle c_{i} c_{j}\right\rangle$ obviously vanish $(\kappa=0)$, and the eigenvalues $f_{\nu}$ of the generalized one body density matrix $\rho^{\text {qsp }}$ reduce to those of the one body density matrix $\rho^{\mathrm{sp}}$, implying $S^{\mathrm{sp}}=S^{\mathrm{qsp}}$.

\section{B. Mixed states and analytic evaluation of the concurrence}

The fermionic concurrence for mixed states can be defined by the convex roof extension of Eq. (43). For twofermion states an explicit expression was derived in [9]. We will here extend this expression to the present general states (see also 22]). Let

$$
\rho=\sum_{k} \lambda_{k}\left|\Psi_{k}\right\rangle\left\langle\Psi_{k}\right|
$$

be a mixed state with eigenvectors $\left|\Psi_{k}\right\rangle$ and eigenvalues $\lambda_{k}$, with $\lambda_{k}>0$ for $k=1 \ldots, r$ and $r \leq 8$ the rank of $\rho$. We will assume that all $\left|\Psi_{k}\right\rangle$ have the same number parity, such that they are of the form (38) or (54), i.e., $\left|\Psi_{k}\right\rangle=\sum_{i=1}^{4} \alpha_{k i} c_{i}^{\dagger}|0\rangle+\bar{\beta}_{k i} c_{i}|\overline{0}\rangle$ in the odd case. Every convex decomposition $\rho=\sum_{j=1}^{r^{\prime}} p_{j}\left|\Phi_{j}\right\rangle\left\langle\Phi_{j}\right|$ can be obtained from these eigenvectors through a $r^{\prime} \times r$ matrix $U$ with orthonormal columns $\left(U^{\dagger} U=I_{r}\right)$ such that $\sqrt{p_{j}}\left|\Phi_{j}\right\rangle=\sum_{k=1}^{r} U_{j k} \sqrt{\lambda_{k}}\left|\Psi_{k}\right\rangle$. Note that the states $\left|\Phi_{j}\right\rangle$ are normalized, so that $p_{j}=\sum_{k=1}^{r} \lambda_{k}\left|U_{j k}\right|^{2}$.

The average fermionic concurrence (generalized Slater measure) of such decomposition is

$$
\begin{aligned}
\left\langle C\left(\left\{p_{j},\left|\Phi_{j}\right\rangle\right\}\right)\right\rangle & =\sum_{j} p_{j} C\left(\left|\Phi_{j}\right\rangle\right)=\sum_{j} p_{j}\left|\left\langle\tilde{\Phi}_{j} \mid \Phi_{j}\right\rangle\right| \\
& =\sum_{j}\left|\sum_{k, l} U_{j k} U_{j l} \sqrt{\lambda_{k} \lambda_{l}}\left\langle\tilde{\Psi}_{k} \mid \Psi_{l}\right\rangle\right|
\end{aligned}
$$

The matrix $C$ of elements

$$
C_{k l}=\sqrt{\lambda_{k} \lambda_{l}}\left\langle\tilde{\Psi}_{k} \mid \Psi_{l}\right\rangle=\sqrt{\lambda_{k} \lambda_{l}}\left(\boldsymbol{\beta}_{l}^{\dagger} \boldsymbol{\alpha}_{k}+\boldsymbol{\beta}_{k}^{\dagger} \boldsymbol{\alpha}_{l}\right),
$$


is complex symmetric. Therefore, it admits a decomposition of the form [9] $C=V D V^{T}$, where $V$ is a unitary matrix and $D$ is a real diagonal matrix whose diagonal elements $d_{k} \geq 0$ are the square root of the eigenvalues of $C C^{\dagger}=C \bar{C}$, sorted in descending order. Defining $S=U V$, Eq. [61) then reads

$$
\left\langle C\left(\left\{p_{j},\left|\Phi_{j}\right\rangle\right\}\right)\right\rangle=\sum_{j}\left|\sum_{k} S_{j k}^{2} d_{k}\right| .
$$

Since $\sum_{j}\left|\sum_{k} S_{j k}^{2} d_{k}\right| \geq \sum_{j}\left(d_{1}\left|S_{j 1}^{2}\right|-\sum_{k \geq 2}\left|S_{j k}^{2}\right| d_{k}\right)=$ $d_{1}-\sum_{k \geq 2} d_{k}$, a necessary condition for the "separability" of $\rho$, i.e., for $\rho$ to be a convex mixture of Slater determinants with the same number parity, is

$$
d_{1} \leq \sum_{k \geq 2} d_{k}
$$

As in the case of two fermion states, we will now show, following the scheme of [9], that this is also a sufficient condition for separability. Indeed, from 63 it is seen that $\rho$ is separable if there is a matrix $S$ with orthonormal columns such that for every $j$,

$$
\left|\sum_{k=1}^{r} d_{k} S_{j k}^{2}\right|=0
$$

Now, provided condition [64) is fulfilled, there are always phases $\theta_{k}, k=2, . ., r$ such that $d_{1}=\left|\sum_{k=2}^{r} d_{k} e^{i \theta_{k}}\right|$. Then a matrix with elements $S_{j k}=\frac{e^{i\left(\theta_{k}+\mu_{j k} \pi\right)}}{\sqrt{r^{\prime}}}$, where $\mu_{j k}=0,1$ and $\theta_{1}=0$, will give the desired decomposition if the signs $e^{i \mu_{j k} \pi}$ can be arranged such that the condition $S^{\dagger} S=I_{r}$ is satisfied. This can be ensured by taking $r^{\prime}=2$ if $r=2, r^{\prime}=4$ if $r=3,4$ [9] and $r^{\prime}=8$ if $5 \leq r \leq 8$, where we can set $\mu_{j 1}=0 \forall j$ and $\left(\mu_{1 k}, \ldots, \mu_{8 k}\right)$ as $(0,0,0,0,1,1,1,1),(0,0,1,1,0,0,1,1)$, $(0,0,1,1,1,1,0,0),(0,1,0,1,0,1,0,1),(0,1,0,1,1,0,1,0)$, $(0,1,1,0,0,1,1,0),(0,1,1,0,1,0,0,1)$ for $k=2, \ldots, 8$. This completes the proof.

On the other hand, if condition (64) does not hold, the average (63) is not smaller than $d_{1}-\sum_{k=2}^{r} d_{k}$. This lower bound may be achieved with the same construction used above, choosing $\theta_{k}=\pi / 2$ for $k \geq 2$. Then, the minimizing decomposition is that where all the components have the same concurrence, which is the concurrence of the state $\rho$,

$C(\rho)=\operatorname{Min}_{\left\{p_{j},\left|\Phi_{j}\right\rangle\right\}} \sum_{j} p_{j} C\left(\left|\Phi_{j}\right\rangle\right)=\operatorname{Max}\left[d_{1}-\sum_{k=2}^{r} d_{k}, 0\right]$.

Using the dualization matrix (53) we may also obtain the eigenvalues $d_{k}$ as those of

$$
R=\sqrt{\rho^{1 / 2} T \rho^{*} T \rho^{1 / 2}}
$$

where $\rho^{*}$ means conjugation in the basis where $T$ takes the form (53).
Once $C$ is obtained, we can evaluate the convex roof extension (37) of $S^{\text {qsp }}$ as

$$
E^{\mathrm{qsp}}(\rho)=4 h\left(\frac{1+\sqrt{1-C^{2}(\rho)}}{2}\right)
$$

in the same way as in the two-qubit case 26, since for pure states we have similarly $S^{\text {qsp }}=4 h\left(\frac{1+\sqrt{1-C^{2}(|\Psi\rangle)}}{2}\right)$ (Eq. (44)), which is a convex increasing function of $C(|\Psi\rangle)$. The quantity $\frac{1+\sqrt{1-C^{2}(\rho)}}{2}$ is also the maximum fidelity between $\rho$ and a convex mixture of gaussian states, as shown in 22] with a different treatment based on group-theoretical methods.

A general mixed state $\rho$ satisfying $[\rho, P]=0$ will be a convex mixture of pure states with even and odd number parity. It can be written as a convex mixture of even and odd parts, i.e.,

$$
\rho=p_{+} \rho_{+}+p_{-} \rho_{-},
$$

where $\rho_{ \pm}=\frac{1}{2 p_{ \pm}}(1 \pm P) \rho$ are the even and odd components of $\rho$ and $p_{ \pm}=\operatorname{Tr} \rho(1 \pm P) / 2$ the corresponding probabilities. Since we just consider pure states with definite number parity, for the general mixed states [69) we may just take $E^{\mathrm{qsp}}(\rho)=p_{+} E^{\mathrm{qsp}}\left(\rho_{+}\right)+p_{-} E^{\mathrm{qsp}}\left(\rho_{-}\right)$, with $E^{\mathrm{qsp}}\left(\rho_{ \pm}\right)$evaluated with Eqs. (66) and (68).

As illustration, we consider a definite parity mixture of a maximally entangled state $|\Psi\rangle(C(|\Psi\rangle)=1)$ with the fully mixed state,

$$
\rho=p|\Psi\rangle\langle\Psi|+(1-p) I_{8} / 8
$$

where $0 \leq p \leq 1$. In the odd parity case, $|\Psi\rangle$ can be written in the form

$$
|\Psi\rangle=\frac{1}{\sqrt{2}}\left(c_{1}^{\dagger}|0\rangle+c_{1}|\overline{0}\rangle\right)=\frac{1}{\sqrt{2}}\left(c_{1}^{\dagger}+c_{2}^{\dagger} c_{3}^{\dagger} c_{4}^{\dagger}\right)|0\rangle,
$$

whereas in the even parity case we can take $|\Psi\rangle=$ $\frac{1}{\sqrt{2}}(|0\rangle+|\overline{0}\rangle)$ or $\frac{1}{\sqrt{2}}\left(c_{1}^{\dagger} c_{2}^{\dagger}+c_{3}^{\dagger} c_{4}^{\dagger}\right)|0\rangle$. A direct calculation using (66) leads to

$$
C(\rho)=\operatorname{Max}\left[\frac{7 p-3}{4}, 0\right],
$$

indicating entanglement for $p>3 / 7$, i.e. $q>1 / 2$, where $q=\langle\Psi|\rho| \Psi\rangle=p+(1-p) / 8$ is the total weight of $|\Psi\rangle$. A similar calculation but considering just twofermion states, $\rho_{2}=p|\Psi\rangle\langle\Psi|+(1-p) I_{6} / 6$, leads instead to $C\left(\rho_{2}\right)=\operatorname{Max}\left[\frac{5 p-2}{3}, 0\right]$, implying entanglement above a slightly smaller value of $p(p>2 / 5$, entailing again $q=p+(1-p) / 6>1 / 2)$, with $C\left(\rho_{2}\right)>C(\rho)$ for $p \in(2 / 5,1)$. As in the two-qubit case, the existence of a finite threshold probability $p$ for non-zero $C$ and hence $E^{\mathrm{qsp}}$, implies a finite limit temperature for entanglement $T_{L}$ if $\rho$ represents a thermal state $\left(\frac{q}{(1-p) / 8} \propto e^{-\beta\left(E_{0}-E_{1}\right)}\right.$, with $E_{0}$ the energy of $|\Psi\rangle$ and $E_{1}>E_{0}$ that of remaining 7 levels), which is larger in the second canonical case. 


\section{CONCLUSIONS}

We have presented a general consistent formalism for describing entanglement-like correlations in general fermion states with no definite fermion number yet fixed number parity. We have first defined a single level entanglement entropy that quantifies the entanglement between a single-particle mode and its orthogonal complement, through the definition of suitable reduced states for such a partition of a given basis of the single-particle space. The sum over all sp modes of this entropy, $S_{c}$, can be taken as a measure of the total entanglement of the system with respect to this basis, and its minimum over all sp bases, $S^{\mathrm{sp}}$, was shown to be a function of the one-body density matrix, being then invariant with respect to unitary transformations in the single-particle space. Moreover, if minimization is extended over all quasiparticle basis, the resulting entanglement entropy, $S^{\mathrm{qsp}}$, is a function of the generalized one-body density matrix, remaining therefore invariant under general Bogoliubov transformations. Such entropy vanishes iff there is a single particle or quasiparticle basis in which every level is separable from its orthogonal complement, i.e., iff each of these levels is either empty or occupied. These entanglement entropies satisfy the inequality chain $S_{c} \geq S^{\mathrm{sp}} \geq S^{\mathrm{qsp}}$. The convex roof extension of $S^{\mathrm{qsp}}$ was also introduced, its vanishing rigorously identifying "classically" correlated mixed fermion states which can be expressed as convex mixtures of pure states or quasiparticle vacua, like those emerging at sufficiently high temperatures in interacting many-fermion systems through approaches like the SPA.

In the case of fermion systems with four single particle levels, a fermionic analog of the two-qubit pure state concurrence was defined in terms of $\rho^{\mathrm{qsp}}$, which reduces to the Slater correlation measure defined in [9, 11] for twofermion states. The eigenvalues of the generalized onebody density matrix, which are four-fold degenerate, can be written as functions of this concurrence and consequently, the entanglement entropy $S^{\mathrm{qsp}}$ is related to the fermionic concurrence by an expression analogous to that of the two-qubit case. This result suggests that "particle entanglement" may be seen as a minimum "mode entanglement". For mixed states with fixed number parity of this system, an explicit expression for the fermionic concurrence, defined as the convex roof extension of the pure state concurrence, was derived, in complete analogy to the two-qubit case, which generalizes the result of 9, 11] and provides a closed analytic expression for the convex roof extension of $S^{\mathrm{qsp}}$.

\section{ACKNOWLEDGMENTS}

The authors acknowledge support from CONICET (NG) and CIC (RR) of Argentina.

\section{Appendix A: Quasiparticle vacuum}

According to Thouless theorem [36] the vacuum $\left|0_{\boldsymbol{a}}\right\rangle$ of the quasiparticle fermion operators (19) is given, if Det $U \neq 0$, by 24.

$$
\begin{aligned}
\left|0_{\boldsymbol{a}}\right\rangle & =\gamma \exp \left[\frac{1}{2} \sum_{i, j} T_{i j} c_{i}^{\dagger} c_{j}^{\dagger}\right]|0\rangle \\
& =\gamma\left[1+\frac{1}{2} \sum_{i, j} T_{i j} c_{i}^{\dagger} c_{j}^{\dagger}+\ldots\right]|0\rangle,
\end{aligned}
$$

where $\gamma=\sqrt{|\operatorname{Det} U|}$ and $T=-U^{-1} V$ is an antisymmetric matrix, with $|0\rangle$ the vacuum of the $c_{j}$ operators. Eq. (A1) can be verified by directly applying $a_{\nu}$ to A1 (if Det $U=0,\left|0_{a}\right\rangle$ can be obtained by applying additional creation operators $c_{j}^{\dagger}$ to Eq. (A1).

If $|\Psi\rangle=\left|0_{\boldsymbol{a}}\right\rangle$, then $f_{\nu}=\left\langle 0_{\boldsymbol{a}}\left|a_{\nu}^{\dagger} a_{\nu}\right| 0_{\boldsymbol{a}}\right\rangle=0 \forall \nu$, imply$\operatorname{ing} S^{\mathrm{qsp}}=0$. However, it is easy to see that

$$
\rho^{\mathrm{sp}}=1-\left\langle 0_{\boldsymbol{a}}\left|\boldsymbol{c} \boldsymbol{c}^{\dagger}\right| 0_{\boldsymbol{a}}\right\rangle=V V^{\dagger},
$$

implying $S^{\mathrm{sp}}>0$ if $V \neq 0$. The eigenvalues $p_{k}$ of $\rho^{\mathrm{sp}}$ are then just the square of the singular values of $V$. The state $|\Psi\rangle$ appears, therefore, mixed at the sp level, reflecting that it cannot be written as a Slater determinant in operators of the form (13).
[1] M.A. Nielsen and I.L. Chuang, Quantum Computation and Quantum Information (Cambridge Univ. Press, Cambridge, UK, 2000).

[2] C.H. Bennett et al, Phys. Rev. Lett. 70, 1895 (1993).

[3] R. Josza and N. Linden, Proc. R. Soc. London, Sect. A 459, 2011 (2003); G. Vidal, Phys. Rev. Lett. 91, 147902 (2003).

[4] R. Horodecki, P. Horodecki, M. Horodecki and K. Horodecki, Rev. Mod. Phys. 81, 865 (2009).

[5] G. Vidal, J.I. Latorre, E. Rico, and A. Kitaev, Phys. Rev. Lett. 90, 227902 (2003).

[6] L. Amico, R. Fazio, A. Osterloh, and V. Vedral, Rev. Mod. Phys. 80, 517 (2008).

[7] J. Eisert, M. Cramer, and M.B. Plenio, Rev. Mod. Phys.
82, 277 (2010).

[8] A. Peres, Quantum Theory,Concepts and Methods (Kluwer Academic, The Netherlands, 1995).

[9] J. Schliemann, J.I. Cirac, M. Kus, M. Lewenstein, D. Loss, Phys. Rev. A 64, 022303 (2001).

[10] J. Schliemann, D. Loss, and A.H. MacDonald, Phys. Rev. B 63, 085311 (2001).

[11] K. Eckert, J. Schliemann, D. Bruß and M. Lewenstein, Ann. Phys. 299, 88 (2002).

[12] P. Zanardi, Phys. Rev. A 65, 042101 (2002).

[13] Y. Shi, Phys. Rev. A 67, 024301 (2003).

[14] N. Friis, A.R. Lee, and D.E. Bruschi, Phys. Rev. A 87, 022338 (2013).

[15] H.M. Wiseman and J.A. Vaccaro, Phys. Rev. Lett. 91, 
097902 (2003).

[16] G.C. Ghirardi and L. Marinatto Phys. Rev. A 70, 012109 (2004).

[17] A. Plastino, D. Manzano, and J. Dehesa, Europhys. Lett. 86, 20005 (2009).

[18] F. Iemini and R.O. Vianna, Phys. Rev. A 87, 022327 (2013); F. Iemini, T.O. Maciel, and R.O. Vianna, Phys. Rev. B 92, 075423 (2015).

[19] F. Benatti, R. Floreanini, U. Marzolino, Phys. Rev. A 89, 032326 (2014).

[20] X.M. Puspus, K.H. Villegas, F.N.C. Paraan, Phys. Rev. B 90, 155123 (2014).

[21] C.V. Kraus, M.M. Wolf, J.I. Cirac, G. Giedke, Phys. Rev. A 79, 012306 (2009).

[22] M. Oszmaniec, J. Gutt, M. Kuś, Phys. Rev. A 90, 020302(R) (2014); M. Oszmaniec, M. Kuś, Phys. Rev. A 90, 010302(R) (2014); Phys. Rev. A 88, 0523328 (2013).

[23] G. Sárosi, P. Lévay, J. Phys. A 47, 115304 (2014); Phys. Rev. A 90, 052303 (2014); Phys. Rev. A 89, 042310 (2014).

[24] P. Ring, P. Schuck, The Nuclear Many-Body Problem (Springer, Berlin, Germany, 1980).

[25] N. Friis, arXiv:1502.04476

[26] S. Hill and W.K. Wootters, Phys. Rev. Lett. 78, 5022
(1997); W.K. Wootters, Phys. Rev. Lett. 80, 2245 (1998).

[27] N. Canosa, R. Rossignoli, Phys. Rev. Lett. 88, 170401 (2002).

[28] N. Gigena, R. Rossignoli, Phys. Rev. A 90, 042318 (2014).

[29] R. Bhatia, Matrix Analysis, Springer, NY (1997).

[30] R.F. Werner, Phys. Rev. A 40, 4277 (1989).

[31] C.H. Bennett, D.P. DiVincenzo, J.A. Smolin, W.K. Wootters, Phys. Rev. A 54, 3824 (1996).

[32] B. Mühlschlegel, D.J. Scalapino, and R. Denton, Phys. Rev. B 6, 1767 (1972).

[33] Y. Alhassid, J. Zingman, Phys. Rev. C 30, 684 (1984); P. Arve, G. Bertsch, B. Laurizten and G. Puddu, Ann. Phys. (N.Y.) 183, 309 (1988). R. Rossignoli, A. Ansari, P. Ring, Phys. Rev. Lett. 70, 1061 (1993); R. Rossignoli, N. Canosa, P. Ring, Phys. Rev. Lett. 80, 1853 (1998).

[34] J. Hubbard, Phys. Rev. Lett. 3, 77 (1959); R.L. Stratonovich, Dokl. Akad. Nauk. SSSR 115, 1097 (1957) [Soviet Phys. Doklady 2, 416 (1957)].

[35] F. de Melo, P. Ćwikliński, B.M. Terhal, New J. Phys. 15, 013015 (2013).

[36] D.J. Thouless, Nucl. Phys. 21, 225 (1960). 